\title{
BMJ Open 10-year trend of tooth loss and associated factors in a Japanese population-based longitudinal study
} Michiko Furuta (i) , ${ }^{1}$ Kenji Takeuchi, ${ }^{1,2}$ Toru Takeshita, ${ }^{1,3}$ Yukie Shibata, ${ }^{1}$
Shino Suma, ${ }^{1}$ Shinya Kageyama, ${ }^{1}$ Mikari Asakawa, ${ }^{1}$ Jun Hata, ${ }^{4}$ Daigo Yoshida,
Yoshihiro Shimazaki, ${ }^{5}$ Toshiharu Ninomiya, ${ }^{4}$ Yoshihisa Yamashita (D) ${ }^{1}$
To cite: Furuta M, Takeuchi K, Takeshita T, et al. 10-year trend of tooth loss and associated factors in a Japanese population-based longitudinal study. BMJ Open 2021;11:e048114. doi:10.1136/ bmjopen-2020-048114

- Prepublication history and additional supplemental material for this paper are available online. To view these files, please visit the journal online (http://dx.doi.org/10.1136/ bmjopen-2020-048114)

Received 18 December 2020 Accepted 30 July 2021
Check for updates

(C) Author(s) (or their employer(s)) 2021. Re-use permitted under CC BY-NC. No commercial re-use. See rights and permissions. Published by BMJ.

For numbered affiliations see end of article.

Correspondence to Dr Yoshihisa Yamashita; yoshi@dent.kyushu-u.ac.jp

\section{ABSTRACT}

Objectives We examined the trend in the number of missing teeth in a Japanese community over a 10-year period and the potential associated explanatory factors. Design Prospective cohort study.

Setting A population-based study conducted in 2007, 2012 and 2017 in Japan (Hisayama Study).

Participants Residents of a Japanese community aged 40-79 years undergoing dental examination in 2007 $(n=2665), 2012(n=2325)$ and $2017(n=2285)$.

Outcome measures The number of missing teeth, periodontal condition, dental caries experience, dental plaque index and oral health behaviours were evaluated each year. The longitudinal analysis of variation in these factors were assessed using mixed models.

Results The age-adjusted and sex-adjusted mean number of missing teeth decreased with time $(6.80$ in 2007, 6.01 in 2012 and 4.99 in 2017). The mean clinical attachment level (CAL), prevalence of periodontitis and dental plaque index decreased over the study period, while dental caries experience slightly increased. The level of oral health behaviour increased over time. Poisson mixed models showed that changes in mean CAL and dental caries experience were positively associated with the change in the number of missing teeth over time. Linear mixed models showed that changes in dental plaque index and no regular dental visit were positively associated with changes in mean CAL.

Conclusions These findings suggest that a decreasing trend regarding the number of missing teeth in Japan might be associated with improvements in the periodontal condition due to changes in oral hygiene level and oral health behaviour.

\section{INTRODUCTION}

The number of teeth is a key indicator of the oral health condition of populations, especially in senior adults. The age-standardised prevalence of edentulism in the global population decreased from $4.4 \%$ in 1990 to $2.4 \%$ in 2010. The incidence rate of edentulous individuals reduced from 374 cases to 205 cases per 100000 person-years over the last two decades. ${ }^{1}$ Decreased severe tooth loss over the past two decades suggests that global oral
Strengths and limitations of this study

- The majority of studies on tooth loss trends have focused on the complete loss of all teeth.

- This study evaluated the number of missing teeth as gradual tooth loss.

- This is the first study to comprehensively investigate the changes in the number of missing teeth, periodontal condition, dental plaque and oral health behaviours in community-dwelling adults over a long time period in Japan.

- The dental examination participation rate decreased in 2012 and 2017, which suggests that the participants of these years might be less representative of the general population.

health is constantly improving. This updated information regarding changes in oral health may help in understanding the needs of the population and facilitating the formulation of policies for further improvement of oral health. Additionally, trends in tooth loss are necessary for estimating population levels of oral health, planning dental health services and for evaluating global oral health goals at the population level. The majority of studies in trends in tooth loss have focused on the complete loss of all teeth. ${ }^{1-5}$ Although it is easy to detect edentulous dentition, this outcome fails to evaluate many health factors associated with gradual tooth loss. Analysis of earlier stages of tooth loss and their associated factors may be important for oral health.

The Japanese Ministry of Health, Labor and Welfare and the Japanese Dental Association have carried out a social campaign ('8020 movement') since 1991. This programme educates the population about the benefits of retaining 20 or more of one's own teeth at the age of 80 , such as maintaining a satisfactory dietary life. Indeed, the presence of at least 20 teeth is enough to assure sufficient masticatory function and aesthetic satisfaction. ${ }^{6}$ 
In part because of the 8020 movement, the findings of a national survey in Japan (Survey of Dental Diseases, SDD) indicate that the proportion of elderly individuals with at least 20 teeth has increased over time. ${ }^{7}$ Interpretation of the SDD requires caution as the sampling methods differ each survey year. ${ }^{8}$ The SDD was conducted every 6 years until 2011 and then every 5 years thereafter. The SDD is carried out at the same time as the National Health and Nutrition Survey (NHNS). The SDD in 2005 was a stratified two-stage cluster sample design to obtain a nationally representative sample of the Japanese population, whereas the SDD in 2011 was a stratified singlestage cluster sample design to obtain an exceptionally expanded sample for comparison across 47 prefectures. $^{9}$ The SDD in 2016 selected the survey areas chosen for the NHNS, whose survey area was chosen for the Population Census of Japan, to obtain a nationally representative sample of the Japanese population. ${ }^{8}$ Because of the different sampling methods in each survey year of the SDD, the trend in tooth loss is difficult to understand. Population-based epidemiological studies are needed to confirm the trend in tooth loss, but there has been no study investigating the trend in tooth loss in Japan other than the SDD, which has its limitations.

Periodontitis and dental caries are considered major causes of tooth loss. Tooth loss reflects not only oral disease but also oral hygiene level and oral health behaviour. ${ }^{10}$ To understand trends in tooth loss, it is necessary to explore changes in factors associated with tooth loss over time. ${ }^{10}$ However, as far as we know, no population-based cohort study in Japan has comprehensively investigated the changes in these factors in a single population for a long time period.

The objective of this study was to examine the trend in tooth loss in a general Japanese community between 2007 and 2017. We also discuss potential explanatory factors for the trends in this community.

\section{METHODS}

\section{Study population}

This study was conducted as part of the Hisayama Study, a population-based prospective study of cardiovascular disease, in the town of Hisayama, a suburb of the Fukuoka metropolitan area in southern Japan. Demographic characteristics of this population, such as age and occupation, were similar to the general Japanese population. ${ }^{11}$ Dental examinations began in 1998 for the residents of Hisayama and have been conducted annually since 2003 with the purpose of early detection and prevention of oral diseases. To date, several studies have been published using data mainly from the dental examinations. ${ }^{12-22}$

The Hisayama Study has established new cohorts for cardiovascular disease at 5 -year intervals. ${ }^{23}$ Dental examinations were conducted in Hisayama residents aged $\geq 40$ years in accordance with the establishment of these cohorts. Dental examinations of all residents were conducted in 2007, 2012 and 2017, whereas in all other years they were provided to only a subset of residents (online supplemental table 1); therefore, this study did not use the data from those years. Dental examinations were conducted at the health centre in Hisayama. Nonindependent older people, including those who required long-term nursing care, could not participate, and the participation rate was lower in the older age groups. Therefore, participants aged $\geq 80$ years might not be representative of the older population; thus, these participants were excluded.

The study population consisted of 2665 participants who were 40-79 years old and received both medical and dental examinations in 2007, representing $70 \%$ of residents in this age group. Similarly, the number of participants was 2325 (57.4\%) in 2012 and 2285 (54.1\%) in 2017. The numbers of participants according to 10-year age groups are shown in online supplemental table 2. Informed written consent was obtained from all participants.

\section{Assessment of oral health condition}

The numbers of missing and present teeth, periodontal condition and oral hygiene status were recorded as indicators of oral health condition. According to modifications made by the WHO in the 5th edition of Oral Health Surveys: Basic Methods, ${ }^{24}$ permanent dentition status of each tooth was determined as a sound tooth with no evidence of treated or untreated caries, a tooth with untreated caries, a filled tooth including a fixed dental prosthesis due to caries or any other reason, an extracted tooth without replacement by a dental prosthesis, a missing tooth replaced by a fixed bridge abutment, denture or implant, or an unerupted tooth. The number of missing teeth was calculated by the sum of missing teeth that were not replaced by dental prostheses or replaced by a fixed bridge abutment, denture or implant. When the third molar was absent, we did not include it as a missing tooth because we did not identify whether it was extracted or simply not erupted. A periodontal examination was performed on all teeth except the third molars at two sites (mesiobuccal and midbuccal), following the National Health and Nutrition Examination Survey (NHANES) III method. Additionally, probing pocket depth (PPD) and clinical attachment level (CAL) were assessed. The periodontal examination has been described previously in more detail. ${ }^{15}$ The mean PPD and the mean CAL were calculated as the sum of the maximum PPD or CAL per tooth, divided by the number of teeth present in each individual. Periodontitis was defined by the 1999 and 2018 classification. ${ }^{25-28}$ In the 1999 classification, moderate periodontitis was defined as the presence of two or more interproximal sites with CAL $\geq 4 \mathrm{~mm}$ (not on the same tooth) or two or more interproximal sites with $\mathrm{PPD} \geq 5 \mathrm{~mm}$ (not on the same tooth). ${ }^{25}$ Severe periodontitis was defined as the presence of two or more interproximal sites with CAL $\geq 6 \mathrm{~mm}$ (not on the same tooth) or one or more interproximal sites with $\mathrm{PPD} \geq 5 \mathrm{~mm}$ (not on the same tooth). ${ }^{25}$ Periodontitis 
in the 1999 classification was dichotomised as either no/ mild periodontitis or moderate/severe periodontitis. In the 2018 classification, periodontitis was defined as interdental clinical attachment loss at $\geq 2$ non-adjacent teeth or buccal or oral clinical attachment loss $\geq 3 \mathrm{~mm}$ with pocketing $\geq 3 \mathrm{~mm}$ on $\geq 2$ teeth. Regarding the staging of periodontitis, clinical attachment losses of $1-2 \mathrm{~mm}, 3-4 \mathrm{~mm}$ and $\geq 5 \mathrm{~mm}$ were defined as stages I, II and III, respectively. By assessing maximum PPD (stage I, PPD $\leq 4 \mathrm{~mm}$; stage II, PPD $\leq 5 \mathrm{~mm}$; stage III, $\mathrm{PPD} \geq 6 \mathrm{~mm}$ ), stage-shifting was possible only when the stage increased. ${ }^{28}$ This study did not examine the reasons for missing teeth. Therefore, stage shifting complexity factors, including the present number of teeth, were considered to discriminate between stages III and IV. Less than 20 teeth was classified as stage IV. ${ }^{28}$ Periodontitis in the 2018 classification was dichotomised as either no/stage I to II periodontitis or stage III to IV periodontitis.

In the 2007 survey, a total of nine trained dentists assessed the periodontal condition. The inter-examiner agreement for PPD measurements within $\pm 1 \mathrm{~mm}$ between eight research examiners and the other examiner (YShim) as the gold-standard examiner was very high (kappa >0.8) ${ }^{15}$ In the 2012 survey, the inter-examiner agreement between 10 research examiners and one examiner (YShim) as the gold-standard examiner ranged between kappa values of $0.80-0.95$ for PPD measurements. In the 2017 survey, one examiner (MF) had a very high inter-examiner agreement with the previous gold-standard examiner (YShim) in the 2007 and 2012 surveys (kappa value was 0.95) and served as a gold-standard examiner. Inter-examiner agreement between the 10 research examiners and the goldstandard examiner (MF) ranged between kappa values of 0.64-1.00 for PPD measurements.

The total number of decayed and filled teeth was used as a measure of assessing dental caries. A dental plaque was assessed by the Silness and Löe plaque index which evaluates both soft debris and mineralised deposits on six selected teeth (tooth numbers: 12, 16, 24, 32, 36 and 44) ${ }^{29}$ The plaque index score is as follows: $(0)$ no plaque; (1) a film of plaque adhering to the free gingival margin and adjacent area of the tooth; (2) moderate accumulation of soft deposits within the gingival pocket, or the tooth and gingival margin; (3) abundance of soft matter within the gingival pocket and/or on the tooth and gingival margin. ${ }^{29}$ The total scores for each tooth were divided by the number of teeth examined to determine the participant's mean plaque index score. ${ }^{29}$

\section{Questionnaire}

Information on participants' toothbrushing frequency, regular dental visits, removal of dental calculus in the past year, current smoking and occupational status were obtained using a self-administered questionnaire. The frequency of tooth brushing was categorised as either one time per day or less or two times per day or more. For dental visits, participants were categorised as those who did or did not regularly visit a dentist for oral care at least once a year. The participants were divided into two groups by habitual smoking status: current smokers and former or non-smokers. Occupational status was classified into three categories: clerical support workers, homemaker, unemployed or retired, and other jobs.

\section{Statistical analysis}

The longitudinal analysis for changes in variables of interest used mixed models (SAS PROC MIXED and GLIMMIX; SAS Institute, Cary, North Carolina).$^{30}$ This method allows for the analysis of correlated observations that are measured repeatedly and the inclusion of participants with different numbers of measurements, giving this approach a distinct advantage over more traditional repeated-measures analytical methods, which remove participants who do not have data at all time points. ${ }^{30}$ The first set of models explored whether survey year was associated with oral condition and health behaviours, adjusted for baseline age and sex. These models estimated changes in oral condition and health behaviours over time. We used linear, Poisson and logistic mixed models based on the distribution of variables. Age-adjusted and sex-adjusted mean values of continuous variable and proportion of participants in categorical variable were estimated using these models. We calculated $\mathrm{p}$ for trend considering the survey year as a continuous variable. In comparing survey years, the survey year in 2007 was used as the reference category.

A second set of models (Poisson mixed model) used the number of missing teeth over time as the outcome. These models explored whether baseline risk factors for tooth loss (periodontal condition, dental caries and oral health behaviours) were associated with the number of missing teeth over time, adjusted for age, sex and baseline number of present teeth and job. The last set of models explored whether the change in risk factors for tooth loss, treated as time-varying variables, was associated with the number of missing teeth over time, adjusted for age, sex, number of present teeth and job. These models estimated the rate ratio of the change in the number of missing teeth per unit change in the independent variable. The additional models explored whether changes in risk factors were associated with the mean CAL or number of decayed and filled teeth over time, adjusted for covariates.

To obtain an understanding of the peculiarity of our study population, the numbers of missing teeth and the proportions of participants with $\geq 20$ present teeth in 2007, 2012 and 2017 were compared with the $\mathrm{SDD}^{7}$ in $2005(\mathrm{n}=3138), 2011 \quad(\mathrm{n}=2908)$ and $2016 \quad(\mathrm{n}=2809)$, respectively. We calculated the number of missing teeth in SDD as 28 minus the number of present teeth in individuals with $\leq 27$ teeth, and as 0 in those with $\geq 28$ teeth because non-erupted wisdom teeth were not treated as missing teeth in the SDD. Using cross-sectional data from the surveys in 2007, 2012 and 2017, comparisons between our study population and the SDD and among survey years in each survey were used a Poisson regression model adjusted for sex. When comparing factors related to oral 
Table 1 Change in oral condition and oral health behaviours among the three surveys

\begin{tabular}{|c|c|c|c|c|}
\hline & 2007 & 2012 & 2017 & P for trend ${ }^{*}$ \\
\hline$n$ & 2665 & 2325 & 2285 & \\
\hline Age†, years & $60.61(0.20)$ & $60.76(0.22)$ & $60.29(0.24)$ & 0.171 \\
\hline Number of missing teeth & $6.80(0.13)$ & $6.01(0.14) \S$ & $4.99(0.16) \S$ & $<0.001$ \\
\hline Number of present teeth & $21.63(0.14)$ & $22.34(0.14) \S$ & $23.37(0.17) \S$ & $<0.001$ \\
\hline Number of decayed and filled teeth & $13.30(0.12)$ & $13.70(0.13)$ & $13.88(0.15) \S$ & $<0.001$ \\
\hline Mean PPDף & $2.39(0.01)$ & $2.16(0.02) \S$ & $1.88(0.02) \S$ & $<0.001$ \\
\hline Mean CALף & $2.83(0.02)$ & $2.46(0.02) \S$ & $2.21(0.02) \S$ & $<0.001$ \\
\hline Moderate/severe periodontitis in the 1999 classificationף, \% & 43.2 & $26.3 \S$ & $17.8 \S$ & $<0.001$ \\
\hline Periodontitis stage III/IV in the 2018 classificationף, \% & 34.9 & $24.5 \S$ & $16.2 \S$ & $<0.001$ \\
\hline Regular dental visit††, \% & 27.3 & $35.9 \S$ & $48.6 \S$ & $<0.001$ \\
\hline
\end{tabular}

Age-adjusted and sex-adjusted mean values (SEs) or proportions in the mixed model.

${ }^{*} \mathrm{P}$ for trend considering the survey year as a continuous variable.

†Unadjusted mean values

łUnadjusted proportions

$\S \mathrm{P}<0.05$ compared each survey in 2012 and 2017 with the survey in 2007 . The survey year in 2007 was used as a reference category.

||Excluding edentulous individuals ( $n=81$ in 2007, $n=50$ in 2012 and $n=24$ in 2017)

${ }^{* *}$ Excluding individuals with missing values ( $n=17$ in 2007, $n=19$ in 2012 and $n=16$ in 2017)

††Excluding individuals with missing values ( $n=50$ in 2007, $n=97$ in 2012 and $n=52$ in 2017)

CAL, clinical attachment level; PPD, probing pocket depth.

health, data from NHNS ${ }^{31}$ in 2004 and 2007 were used. Differences between our study population and NHNS were assessed by the $\chi^{2}$ test.

SAS V.9.4 (SAS Institute) was used to perform all statistical analyses and $\mathrm{p}<0.05$ was considered statistically significant in all analyses.

\section{Patient and public involvement}

Participants were recruited with the help of the Health C\&C Center Hisayama, which provided healthcare services to the residents of Hisayama. None of our participants were involved in the recruitment or conduct of either study that has provided data for this report.

\section{RESULTS}

\section{Changes in oral condition and oral health behaviour}

The age-adjusted and sex-adjusted mean values or frequencies of oral condition and oral health behaviours among the three surveys are shown in table 1 . The average of the number of missing teeth decreased from 2007 and 2017 ( $p$ for trend $<0.001$ ). The mean PPD and CAL decreased from 2007 to 2017, and the prevalence of moderate/severe periodontitis in the 1999 classification and stage III/IV in the 2018 classification decreased over the study period (all $\mathrm{p}$ for trend $<0.001$ ). The mean number of decayed and filled teeth slightly increased from 13.30 in 2007 to 13.88 in 2017. In oral health behaviours, the percentage of participants who brushed their teeth $\geq 2$ times per day and regularly visited a dental clinic increased from 2007 to 2017 (both $\mathrm{p}$ for trend $<0.001$ ).

As shown in figure 1 , the number of missing teeth decreased over survey years in all age groups in our study population, as did the trend in the national survey (all $\mathrm{p}$ for trend $<0.05$, from 2005 to 2016 in national survey and from 2007 to 2017 in Hisayama). The number of missing teeth in our population was compared with that of the national survey in Japan based on different age groups; the number of missing teeth in the 50-59year group in our study population in 2007 was lower than that of the national survey in 2005. The age groups of 60-69 years and 70-79 years in our study population had lower numbers of missing teeth than those of the national survey across all examined years (figure 1). Similarly, the frequencies of participants with $\geq 20$ teeth increased over survey years in all age groups in our study population, as did the trend in the national survey (online supplemental figure 1). We found a higher percentage of individuals with $\geq 20$ teeth in our study population than in the 70-79years group of the national survey across all examined years (online supplemental figure 1).

\section{Baseline risk factors and number of missing teeth over time} The associations of risk factors for tooth loss at baseline with time-varying numbers of missing teeth were shown in online supplemental tables 3 and 4 . The positive associations were seen for mean CAL and PPD, moderate/severe 


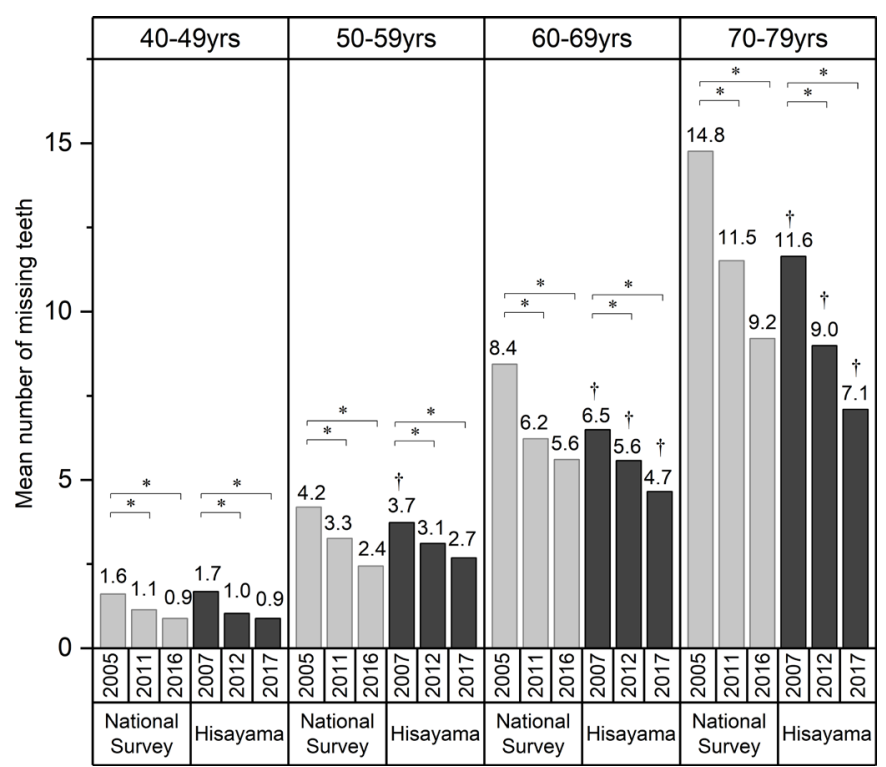

Figure 1 Population-based trends in the sex-adjusted number of missing teeth according to age groups in the national survey and dental examinations in Hisayama. $\mathrm{p}<0.05$, comparing the first year in each survey (2005 in national survey or 2007 in Hisayama) using a Poisson regression model adjusted for sex. ${ }^{\dagger} p<0.05$, comparing Hisayama to the national survey using a Poisson regression model adjusted for sex (national survey in 2005 vs Hisayama in 2007, national survey in 2011 vs Hisayama in 2012 and national survey in 2016 vs Hisayama in 2017).

periodontitis in the 1999 classification and stage III/IV in the 2018 classification, and numbers of decayed and filled teeth after adjusting for age, sex, number of present teeth, and job.

Changes in risk factors and number of missing teeth over time We evaluated whether changes in risk factors for tooth loss influenced the number of missing teeth over time (table 2). The models predicted that increases in mean CAL and number of decayed and filled teeth were positively associated with an increasing number of missing teeth over time, adjusted for age, sex, number of present teeth and job at baseline. No significant associations with number of missing teeth were seen for changes in dental plaque, toothbrushing frequency or no regular dental visits over time.

\section{Factors associated with mean CAL and number of decayed and filled teeth over time}

When we explored factors associated with mean CAL over time, increases in dental plaque and no regular dental visits were associated with increasing mean CAL (table 3). No regular dental visit was inversely associated with the number of decayed and filled teeth over time (table 3).

\section{DISCUSSION}

We found that the number of missing teeth has decreased over 10 years, both in our population as well as that of the national survey. The mixed models showed that increases

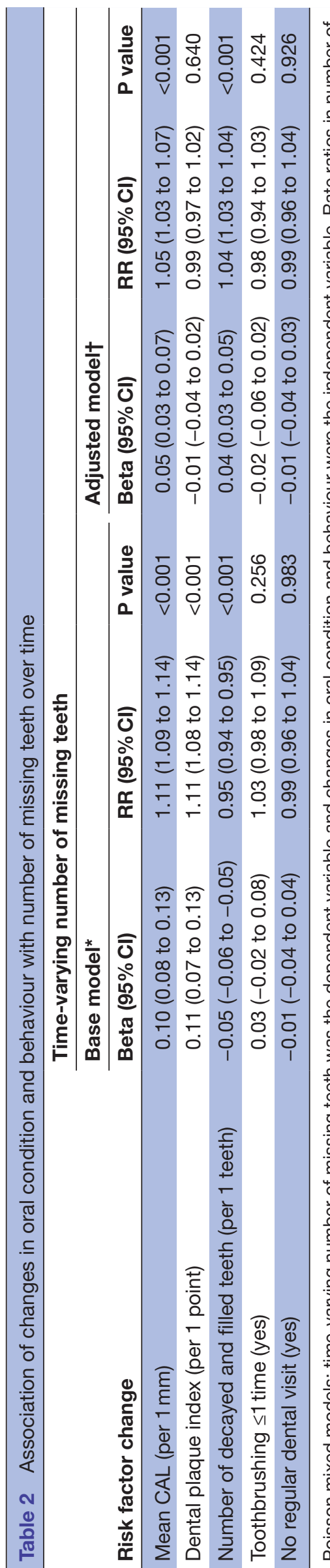


Table 3 Association of changes in dental plaque and oral behaviour with mean CAL or number of decayed and filled teeth over time

\begin{tabular}{|c|c|c|c|c|}
\hline & \multicolumn{2}{|l|}{ Base model* } & \multicolumn{2}{|l|}{ Adjusted model† } \\
\hline & Beta $(95 \% \mathrm{Cl})$ & $P$ value & Beta $(95 \% \mathrm{CI})$ & $P$ value \\
\hline \multicolumn{5}{|l|}{ Time-varying mean CAL } \\
\hline Toothbrushing $\leq 1$ time (yes) & $0.15(0.09$ to 0.20$)$ & $<0.001$ & $0.03(-0.03$ to 0.08$)$ & 0.350 \\
\hline No regular dental visit (yes) & $0.22(0.18$ to 0.27$)$ & $<0.001$ & 0.15 (0.11 to 0.20$)$ & $<0.001$ \\
\hline \multicolumn{5}{|c|}{ Time-varying number of decayed and filled teeth§ } \\
\hline Dental plaque index (per 1 point) & $-0.03(-0.45$ to -0.12$)$ & $<0.001$ & 0.17 (0.01 to 0.33$)$ & 0.034 \\
\hline Toothbrushing $\leq 1$ time (yes) & $-0.49(-0.75$ to -0.23$)$ & $<0.001$ & $-0.17(-0.41$ to 0.08$)$ & 0.177 \\
\hline No regular dental visit (yes) & $-0.39(-0.59$ to -0.19$)$ & $<0.001$ & $-0.24(-0.42$ to -0.06$)$ & 0.011 \\
\hline
\end{tabular}

*Base model; linear mixed model adjusted for age and sex.

†Adjusted model included all independent variables (time-varying variables), age, sex, number of present teeth and job at baseline.

‡Linear mixed models; time-varying mean CAL was the dependent variable and changes in dental plaque and oral health behaviours were the independent variable.

§Linear mixed models; time-varying number of decayed and filled teeth was the dependent variable and changes in dental plaque and oral health behaviours were the independent variable.

CAL, clinical attachment level.

in mean CAL and number of decayed and filled teeth were associated with an increasing number of missing teeth. The reduction in tooth loss in the study period may be explained by fewer teeth being extracted because of periodontitis. We support this conclusion with the findings that periodontal condition, oral hygiene level and oral health behaviours showed improvement over time, while dental caries experience increased (table 1). We found the positive association of dental plaque and regular dental visit with periodontal condition (table 3). Additionally, we observed a decline in smoking prevalence in our study population, particularly among men. ${ }^{32}$ These findings suggest that improvement in health behaviours, such as toothbrushing, regular dental visits and smoking, leads to improved oral hygiene, which subsequently reduces the prevalence of periodontitis.

Consistent with our study population, the national survey showed a significant decrease in the number of missing teeth over 10 years. This finding suggests that dental health services in Japan have been effective in preventing tooth loss. In addition, we found that residents of Hisayama in the age groups of 60-69 years and 70-79 years had lower numbers of missing teeth when compared with those same groups in the national survey. A lower number of missing teeth in the town of Hisayama than in the national survey may be attributed to the town's health policy, which has incorporated dental examinations in the regular health check-up since 1998. Additionally, the study centre and town office in Hisayama provide free health examinations, counselling and educational health services. Since approximately $50 \%-70 \%$ of town residents participate in the annual heath check-up, this health policy enables residents to be aware of their own oral condition and to modify oral health behaviours (eg, toothbrushing frequency, use of dental floss and interdental brushing and regular dental visits) before progressive oral disease. Certain risk factors are shared between oral disease and several chronic diseases, include smoking, poor diet and stress. ${ }^{33}$ Performing simultaneous health check-ups and dental examinations may therefore be effective for preventing tooth loss by controlling common risk factors for chronic diseases and oral disease (ie, common risk factor approach).$^{33}$ The Ministry of Health, Labor and Welfare in Japan provides a national healthcare programme, which includes an annual health check-up and health guidance in the workplace and municipality ${ }^{34}$; however, dental examinations are not included. On the basis of the common risk factor approach, further improvement in oral health could be achieved at the national level by adding dental examinations for the prevention of oral disease to the healthcare programme.

The differences in the numbers of missing teeth between our study and that of the national survey may be partly explained by different levels of oral health behaviour. We compared health behaviours between our population and that of the national survey and found that the percentage of participants who were 70-79 years old who had dental calculus removed in the past year was higher in our population in 2007 than that of the national survey (online supplemental table 5). Additionally, the percentage of individuals with untreated dental caries was lower in all age groups in our population compared with that of the national survey (online supplemental table 5). Immediately after dental examination, the residents of Hisayama were informed about their disease status. Understanding of the seriousness of periodontitis and the benefits of behavioural change is associated with a greater likelihood of behaviour change. ${ }^{35}$ We could not compare regular dental visits between our population and that in 
the national survey because the national survey did not collect data on regular dental visits. It was considered that the residents of Hisayama were more likely to visit a dental clinic to prevent aggravation of periodontitis and receive treatment for dental caries after receiving dental examinations.

In regard to dental caries, the numbers of decayed and filled teeth along with regular dental visit tended to increase over the follow-up period (table 1). Additionally, no regular dental visit was inversely associated with the numbers of decayed and filled teeth over time (table 3). It is considered that regular dental visit contributes to detect early dental caries lesion and have more opportunities for treatment of dental caries.

This study had several limitations. First, the use of dental floss and interdental brushing was not investigated in the surveys in 2007 and 2012, thus eliminating the ability to study trends on the use of dental floss and interdental brushing. Second, we used a partial mouth assessment to assess the periodontal condition, which did not include an examination of lingual or palatal sites. Our results may potentially underestimate the periodontal condition. Additionally, we could not compare the periodontal condition between our study population and that of the national survey due to different means of periodontal assessment (this study and the national survey used the NHANES III method and a community periodontal index, respectively). Third, the participation rate in dental examinations was $70 \%$ in 2007 and above 50\% in both 2012 and 2017. The Hisayama Study has attempted to examine $70 \%-80 \%$ of all residents aged 40 years or older in each cohort at intervals of 5 years. ${ }^{23}$ Dental examinations in 2007 included residents aged 40 years or older for the first time and many residents were likely to receive dental examinations. After this survey, some residents refused to receive a dental survey in 2012 and 2017 because they had regularly gone to a dental clinic for check-up. The participants in this study might be progressively less representative of the general population over survey years. Finally, this study included the residents of a municipality in Japan. Our sample size may limit the ability to extrapolate our findings to all Japanese people. Caution is warranted to generalise our findings to the rest of the Japanese population.

In conclusion, this study showed that oral health condition improved in a Japanese community from 2007 to 2017, with decreasing tooth loss, a decreasing prevalence of periodontitis, and increasing levels of oral health behaviour. This study indicated changes of tooth loss along with changes in associated risk factors, which will contribute to the exploration of the global trend in tooth loss.

\footnotetext{
Author affiliations

${ }^{1}$ Section of Preventive and Public Health Dentistry, Division of Oral Health, Growth and Development, Faculty of Dental Science, Kyushu University, Fukuoka, Japan ${ }^{2}$ Department of Preventive Medicine, Nagoya University Graduate School of Medicine, Nagoya, Japan

${ }^{3}$ OBT Research Center, Kyushu University, Fukuoka, Japan
}

${ }^{4}$ Department of Epidemiology and Public Health, Graduate School of Medical Sciences, Kyushu University, Fukuoka, Japan

${ }^{5}$ Department of Preventive Dentistry and Dental Public Health, School of Dentistry, Aichi Gakuin University, Nagoya, Japan

Contributors MF, YShim, JH, DY, TN and YY conceived and planned the project. MF and KT conducted statistical analysis. MF, KT, JH, DY, TN and YY interpreted the data and drafted the article. All authors acquired the data, critically reviewed and approved the final manuscript.

Funding This work was supported by JSPS KAKENHI Grant Number 21K10229 from the Ministry of Education, Science, Sports and Culture of Japan, Tokyo, Japan.

Competing interests None declared.

Patient consent for publication Not required.

Ethics approval This study was approved by the Kyushu University Institutional Review Board for Clinical Research (28-31).

Provenance and peer review Not commissioned; externally peer reviewed.

Data availability statement Not applicable. No additional data are available.

Supplemental material This content has been supplied by the author(s). It has not been vetted by BMJ Publishing Group Limited (BMJ) and may not have been peer-reviewed. Any opinions or recommendations discussed are solely those of the author(s) and are not endorsed by BMJ. BMJ disclaims all liability and responsibility arising from any reliance placed on the content. Where the content includes any translated material, BMJ does not warrant the accuracy and reliability of the translations (including but not limited to local regulations, clinical guidelines, terminology, drug names and drug dosages), and is not responsible for any error and/or omissions arising from translation and adaptation or otherwise.

Open access This is an open access article distributed in accordance with the Creative Commons Attribution Non Commercial (CC BY-NC 4.0) license, which permits others to distribute, remix, adapt, build upon this work non-commercially, and license their derivative works on different terms, provided the original work is properly cited, appropriate credit is given, any changes made indicated, and the use is non-commercial. See: http://creativecommons.org/licenses/by-nc/4.0/.

ORCID iDs

Michiko Furuta http://orcid.org/0000-0002-7267-857X

Yoshihisa Yamashita http://orcid.org/0000-0002-9664-3689

\section{REFERENCES}

1 Kassebaum NJ, Bernabé E, Dahiya M, et al. Global burden of severe tooth loss: a systematic review and meta-analysis. J Dent Res 2014;93:20s-8.

2 Suominen-Taipale AL, Alanen P, Helenius $\mathrm{H}$, et al. Edentulism among Finnish adults of working age, 1978-1997. Community Dent Oral Epidemiol 1999;27:353-65.

3 Sanders AE, Slade GD, Carter KD, et al. Trends in prevalence of complete tooth loss among Australians, 1979--2002. Aust N Z J Public Health 2004;28:549-54.

4 Wu B, Liang J, Landerman L, et al. Trends of edentulism among middle-aged and older Asian Americans. Am J Public Health 2013;103:e76-82.

5 Slade GD, Akinkugbe AA, Sanders AE. Projections of U.S. Edentulism prevalence following 5 decades of decline. J Dent Res 2014;93:959-65.

6 Elias AC, Sheiham A. The relationship between satisfaction with mouth and number and position of teeth. J Oral Rehabil 1998;25:649-61.

7 Japanese Ministry of Health Labour and Welfare. Survey of dental diseases. Available: http://www.mhlw.go.jp/toukei/list/62-17.html [Accessed 20 Feb 2020].

8 The analysis Committee in survey of dental diseases on Japanese Society for oral health. Report of analysis in survey of dental diseases in 2016 (in Japanese). J Dent Hlth 2018;68:106-13.

9 Ikeda N, Takimoto H, Imai S, et al. Data resource profile: the Japan National health and nutrition survey (NHNS). Int $J$ Epidemiol 2015;44:1842-9.

10 Baelum V, van Palenstein Helderman W, Hugoson A, et al. A global perspective on changes in the burden of caries and periodontitis: implications for dentistry. J Oral Rehabil 2007;34:872-906.

11 Hata J, Ninomiya T, Hirakawa Y, et al. Secular trends in cardiovascular disease and its risk factors in Japanese: half- 
century data from the Hisayama study (1961-2009). Circulation 2013;128:1198-205

12 Saito T, Shimazaki Y, Kiyohara Y, et al. Relationship between obesity, glucose tolerance, and periodontal disease in Japanese women: the Hisayama study. J Periodontal Res 2005;40:346-53.

13 Shimazaki Y, Saito T, Yonemoto K, et al. Relationship of metabolic syndrome to periodontal disease in Japanese women: the Hisayama study. J Dent Res 2007;86:271-5.

14 Saito T, Yamaguchi N, Shimazaki Y, et al. Serum levels of resistin and adiponectin in women with periodontitis: the Hisayama study. J Dent Res 2008;87:319-22.

15 Shimazaki Y, Akifusa S, Takeshita T, et al. Effectiveness of the salivary occult blood test as a screening method for periodontal status. J Periodontol 2011;82:581-7.

16 Furuta M, Shimazaki Y, Takeshita T, et al. Gender differences in the association between metabolic syndrome and periodontal disease: the Hisayama study. J Clin Periodontol 2013;40:743-52.

17 Takeshita T, Matsuo K, Furuta M, et al. Distinct composition of the oral Indigenous microbiota in South Korean and Japanese adults. Sci Rep 2014;4:6990.

18 Takeuchi K, Furuta M, Takeshita T, et al. Risk factors for reduced salivary flow rate in a Japanese population: the Hisayama study. Biomed Res Int 2015;2015:1-7.

19 Takeuchi K, Furuta M, Takeshita T, et al. Serum antibody to Porphyromonas gingivalis and periodontitis progression: the Hisayama study. J Clin Periodontol 2015;42:719-25.

20 Takeshita T, Kageyama S, Furuta M, et al. Bacterial diversity in saliva and oral health-related conditions: the Hisayama study. Sci Rep 2016;6:22164.

21 Furuta M, Takeuchi K, Shimazaki Y, et al. Comparison of the periodontal condition in Korean and Japanese adults: a crosssectional study. BMJ Open 2018;8:bmjopen-2018-024332.

22 Asakawa M, Takeshita T, Furuta M, et al. Tongue microbiota and oral health status in community-dwelling elderly adults. mSphere 2018;3. doi:10.1128/mSphere.00332-18. [Epub ahead of print: 15 08 2018].

23 Ninomiya T. Japanese legacy cohort studies: the Hisayama study. J Epidemiol 2018;28:444-51.
24 World Health Organization. Oral health surveys: basic methods. 5 ed, 2013.

25 Page RC, Eke PI. Case definitions for use in population-based surveillance of periodontitis. J Periodontol 2007;78:1387-99.

26 Eke PI, Page RC, Wei L, et al. Update of the case definitions for population-based surveillance of periodontitis. J Periodontol 2012;83:1449-54.

27 Papapanou PN, Sanz M, Buduneli N, et al. Periodontitis: consensus report of Workgroup 2 of the 2017 world workshop on the classification of periodontal and peri-implant diseases and conditions. J Periodontol 2018;89 Suppl 1:S173-82.

28 Tonetti MS, Greenwell H, Kornman KS. Staging and grading of periodontitis: framework and proposal of a new classification and case definition. J Periodontol 2018;89 Suppl 1:S159-72.

29 Löe $\mathrm{H}$. The gingival index, the plaque index and the retention index systems. J Periodontol 1967;38:610-6.

30 Cnaan A, Laird NM, Slasor P. Using the general linear mixed model to analyse unbalanced repeated measures and longitudinal data. Stat Med 1997;16:2349-80.

31 Japanese Ministory of Health Labour and Welfare. National Health and Nutrition Survey 2020 [cited 2020 February 20]. Available: https://www.mhlw.go.jp/bunya/kenkou/kenkou_eiyou_chousa.html [Accessed 18 Feb 2020].

32 Mukai N, Hata J, Hirakawa Y, et al. Trends in the prevalence of type 2 diabetes and prediabetes in a Japanese community, 1988-2012: the Hisayama study. Diabetol Int 2019;10:198-205.

33 Sheiham A, Watt RG. The common risk factor approach: a rational basis for promoting oral health. Community Dent Oral Epidemiol 2000;28:399-406.

34 Tsushita K, S Hosler A, Miura K, et al. Rationale and descriptive analysis of specific health guidance: the nationwide lifestyle intervention program targeting metabolic syndrome in Japan. $J$ Atheroscler Thromb 2018;25:308-22.

35 Newton JT, Asimakopoulou K. Managing oral hygiene as a risk factor for periodontal disease: a systematic review of psychological approaches to behaviour change for improved plaque control in periodontal management. J Clin Periodontol 2015;42 Supp 16:S36-46. 